\title{
Spontaneous freeze out of dark matter from an early thermal phase transition
}

\author{
Lucien Heurtier $\odot^{*}$ \\ Department of Physics, University of Arizona, Tucson, Arizona 85721, USA \\ Hervé Partouche ${ }^{\dagger}$ \\ CPHT, CNRS, Ecole polytechnique, IP Paris, F-91128 Palaiseau, France
}

(Received 21 December 2019; accepted 31 January 2020; published 19 February 2020)

\begin{abstract}
We propose a new paradigm for the thermal production of dark matter in the early Universe, in which dark-matter particles acquire their mass and freeze out spontaneously from the thermal bath after a dark phase transition takes place. The decoupling arises because the dark-matter particles become suddenly nonrelativistic and not because of any decay channel becoming kinematically close. We propose a minimal scenario in which a scalar and a fermionic dark matter are in thermal equilibrium with the standard-model bath. We compute the finite temperature corrections to the scalar potential and identify a region of the parameter space where the fermionic dark-matter mass spontaneously jumps over the temperature when the dark phase transition happens. We explore the phenomenological implications of such a model in simple cases and show that the annihilation cross section of dark-matter particles has to be larger by more than 1 order of magnitude as compared to the usual constant-mass weakly interacting massive particle scenario in order to accommodate the correct relic abundance. We show that in the spontaneous freeze out regime a $\mathrm{TeV}$-scale fermionic dark matter that annihilates into leptons through $s$-wave processes can be accessible to detection in the near future.
\end{abstract}

DOI: 10.1103/PhysRevD.101.043527

\section{INTRODUCTION}

The nature of dark matter (DM) and the way it is produced in the early Universe stand among the biggest puzzles of modern cosmology. The weakly interacting massive particle (WIMP) paradigm has triggered a lot of attention in the past decades due to its simplicity and to the surprising connection which exists in this framework between the energy scale necessary to produce the correct DM relic abundance and the electroweak scale [1-4]. By construction, WIMP scenarios rely on two major ingredients, which are that (i) the dark-matter particles thermalize with the standard-model (SM) bath at high temperature, and that (ii) the cross section of annihilation of dark-matter particles into visible states is small enough in order to guarantee that the freeze out (FO) mechanism generates a sufficient DM relic abundance in the present Universe. As far as theoretical aspects of the model are concerned, the

\footnotetext{
heurtier@email.arizona.edu

herve.partouche@polytechnique.edu
}

Published by the American Physical Society under the terms of the Creative Commons Attribution 4.0 International license. Further distribution of this work must maintain attribution to the author(s) and the published article's title, journal citation, and DOI. Funded by SCOAP ${ }^{3}$. mass of a WIMP candidate cannot be arbitrarily large. Indeed, too heavy WIMPs, which are required to have a small number density in order to not overclose the Universe, need to annihilate efficiently during the freeze out, demanding their cross section of annihilation to violate unitarity constraints [5]. Moreover, for DM masses larger than $\mathcal{O}(10) \mathrm{GeV}$, vanilla versions of the WIMP in which DM particles annihilate exclusively into nucleons have already been mostly ruled out by dark-matter detection experiments and LHC searches [6-54]. In order to avoid direct-detection constraints, one usually has to either seclude dark matter from the colored sector, weakening drastically constraints from direct-detection experiments [55-57], or build models in which the scattering cross section of DM particles on nucleons is naturally suppressed. Indirect-detection constraints can also be avoided by demanding that the cross section of annihilation of dark-matter particles in the Galaxy is velocity suppressed. In this case the flux of cosmic rays produced by the dark sector is reduced and dark matter is effectively invisible in the present Universe [58-64].

Alternatives to the WIMP paradigm usually assume that dark-matter particles are produced out of equilibrium, either from the decay of a heavy particle (moduli and inflaton) $[65,66]$ or from the feeble annihilation of particles that are thermalized with the standard-model bath [67-72]. In all these different scenarios, the dark-matter mass and the 
masses of its decay or annihilation products are assumed to be constant throughout the Universe evolution. In the WIMP scenario in particular, the calculation of the darkmatter relic density relies on the evaluation of the annihilation cross section of dark matter as a function of masses and couplings, which are assumed to be identical at the time of freeze out and at present time.

In the case of the standard model, however, it is known that the whole mass spectrum is temperature dependent. Indeed, the mass of the SM fermions is given by the vacuum expectation value (vev) of the Brout-Englert-Higgs field. At high temperature, before the electroweak phase transition, thermal corrections to the Higgs scalar potential stabilize the latter at the origin $\langle H\rangle=0$ and the standard model is essentially composed of pure radiation. At low temperature, when the electroweak phase transition takes place, the $U(1) \times S U(2)$ gauge group is spontaneously broken and SM particles acquire masses proportionally to the Higgs vev $\langle H\rangle \neq 0$. Therefore, similarly to the SM particles, the mass spectrum of any thermalized dark sector in which masses originate from the spontaneous breaking of some UV symmetry group is expected to evolve with the temperature.

The possibility that the dark-matter mass might be a time-dependent quantity was studied in the past in the context of variable-mass particles in which dark-matter particles interact with a quintessence field [73-75]. Moreover, the effect of thermal corrections to the potential of a dark scalar was also used in the context of the so-called flip-flop vev mechanism [76,77], super-cool dark matter [78], or forbidden freeze in scenario [79], where a second order phase transition is used to kinematically open or close certain annihilation or decay channels in the early Universe.

In this paper we aim to focus on the very simple case of a thermal fermionic dark matter whose mass is sourced by the vev of a dark scalar. We study how the thermal corrections arising from the contact of this scalar with thermalized particles drive a thermal, second order phase transition in the dark sector. In particular, we identify an interesting region of the parameter space where the phase transition enforces the freeze out to take place before the dark-matter mass reaches its zero-temperature value. We refer to this possibility as a spontaneous freeze out (SFO). We explore in particular to which extent in the SFO case the mass of dark-matter particles at freeze out can differ from its value at present time. We also show that the annihilation cross section of DM particles into SM states required to obtain the correct DM relic abundance can differ by more than 1 order of magnitude from the case of a constant-mass WIMP. Therefore, the SFO scenario is more sensitive to DM searches. It is interesting to note that this paradigm was first encountered in the context of string theory [80] where it is natural to expect moduli fields to couple to matter states and undergo nontrivial phase transitions. In Ref. [81] such a scenario was also briefly considered while discussing the entanglement entropy that might as well affect the decoupling of DM particles in such a context.

The paper is organized as follows: In Sec. II we introduce the model on which we focus throughout this work. In Sec. III we derive the master equations necessary to study the evolution of the mass spectrum with the temperature. In Sec. IV we study the dark-matter freeze out in our model and properly define the paradigm of spontaneous freeze out. In Sec. V we study analytically how the decoupling of dark-matter backreacts on the dynamics of the dark scalar. In Sec. VI we finally specify the way dark-matter particles interact with SM states, compute numerically the DM relic density, and confront our model to existing direct- and indirect-detection constraints. Our conclusions, comments, and perspectives can be found in Sec. VII.

\section{THE MODEL}

Our benchmark model is based on the tree-level Lagrangian density

$$
\mathcal{L}_{\text {tree }}=\mathcal{L}_{\mathrm{SM}}+\mathcal{L}_{\text {dark }}+\mathcal{L}_{\text {int }},
$$

where $\mathcal{L}_{\mathrm{SM}}$ is the SM contribution. The dark sector contains a fermion $\psi$ and a real scalar field $\phi$ charged under a $\mathbb{Z}_{2}$ symmetry $^{1}(\phi \rightarrow-\phi)$. The associated Lagrangian is

$$
\mathcal{L}_{\text {dark }}=i \bar{\psi} \not \partial \psi+\frac{1}{2} \partial_{\mu} \phi \partial^{\mu} \phi-y \phi \bar{\psi} \psi-\mathcal{V}_{\text {tree }}(\phi)+\mathcal{L}_{\text {dark }}^{\text {c.t. }},
$$

where $\mathcal{L}_{\text {dark }}^{\text {c.t. }}$ stands for counterterms to be tuned suitably later on. The Lagrangian $\mathcal{L}_{\text {int }}$, which is specified in Sec. VI, contains only interactions between $\psi, \phi$ and SM fields. The potential $\mathcal{V}_{\text {tree }}(\phi)$ is chosen such that the scalar $\phi$ acquires a nonzero vev and sources a mass term for the dark fermion via the Yukawa interaction,

$$
m_{\psi}(\langle\phi\rangle)=y\langle\phi\rangle .
$$

Defining the potential as

$$
\mathcal{V}_{\text {tree }}(\phi)=-\frac{\mu^{2}}{2} \phi^{2}+\frac{\lambda}{4 !} \phi^{4},
$$

where $\mu$ and $\lambda$ are positive, the tree-level scalar vev and the masses of $\phi$ and $\psi$ are given by

$$
\langle\phi\rangle_{\text {tree }}=\mu \sqrt{\frac{6}{\lambda}}, \quad m_{\phi}^{\text {tree }}=\sqrt{2} \mu, \quad m_{\psi}^{\text {tree }}=y \sqrt{\frac{3}{\lambda}} m_{\phi}^{\text {tree }} .
$$

\footnotetext{
${ }^{1}$ This $\mathbb{Z}_{2}$ symmetry forbids the introduction of a bare mass term for the DM fermion $\psi$, and odd powers of the scalar field, which could, in principle, lead to a first order phase transition.
} 
In the following sections, we show the existence of a region in the parameter space where the dark matter present in the Universe today is composed of fermions $\psi$ only, while the dark scalar particles $\phi$ have decayed into SM states. Indeed, the stability of the fermion $\psi$ is guaranteed by the $\mathbb{Z}_{2}$ symmetry $(\psi \rightarrow-\psi)$ inherent to our Lagrangian. Even if we consider a Dirac fermion $\psi$ in Eq. (2), our results are derived for an arbitrary number $n_{F}$ of fermionic degrees of freedom, e.g., $n_{F}=4$ for a Dirac and $n_{F}=2$ for a Majorana fermion.

\section{THERMAL EFFECTIVE POTENTIAL}

From now on, we assume that the interactions contained in the Lagrangian $\mathcal{L}_{\text {int }}$ are sufficient to maintain the dark sector particles $\psi$ and $\phi$ in thermal equilibrium with the visible sector. At finite temperature, virtual loops of the fields $\psi$ and $\phi$ induce corrections to the scalar potential, which are known to induce a restoration of the vacuum $\mathbb{Z}_{2}$ symmetry at high temperature (see, e.g., Ref. [82] for a review and references therein). Therefore, when thermal loop corrections dominate over the zero-temperature treelevel contribution, perturbation theory breaks down.

To see this explicitly, let us consider the thermal effective potential at one loop,

$\mathcal{V}_{1-\text { toop }}^{\text {th }}(T, \phi)=\mathcal{V}_{\text {tree }}(\phi)+\mathcal{V}_{\mathrm{CW}}(\phi)+\mathcal{V}_{\text {dark }}^{\text {c.t. }}(\phi)+\mathcal{F}(T, \phi)$,

where $\mathcal{V}_{\mathrm{CW}}$ is the zero-temperature Coleman-Weinberg contribution and $\mathcal{F}(T, \phi)$ is the free energy. In dimensional regularization, the UV divergence of $\mathcal{V}_{\mathrm{CW}}$ can be removed by adjusting counterterms $\mathcal{V}_{\text {dark }}^{\text {c.t. }}=-\delta_{\mu} \phi^{2}+\delta_{\lambda} \phi^{4}$, which yield in $\overline{\mathrm{MS}}$ scheme the usual result

$$
\begin{aligned}
\mathcal{V}_{\mathrm{CW}}(\phi)+\mathcal{V}_{\text {dark }}^{\text {c.t. }}(\phi)= & \frac{m_{0}(\phi)^{4}}{64 \pi^{2}}\left[\log \left(\frac{m_{0}(\phi)^{2}}{Q^{2}}\right)-\frac{3}{2}\right] \\
& -n_{F} \frac{m_{\psi}(\phi)^{4}}{64 \pi^{2}}\left[\log \left(\frac{m_{\psi}(\phi)^{2}}{Q^{2}}\right)-\frac{3}{2}\right],
\end{aligned}
$$

where $Q$ is the renormalization energy scale. Hence, $\mu$ and $\lambda$ are running parameters that depend implicitly on $Q$. Moreover, the tree-level mass squared of $\phi$ is defined as

$$
m_{0}(\phi)^{2}=-\mu^{2}+\frac{\lambda}{2} \phi^{2}
$$

which is negative when $\phi^{2}<2 \mu^{2} / \lambda$.

The thermal contribution is the Helmholtz free energy density of a gas comprising one bosonic and $n_{F}$ fermionic degrees of freedom. At one loop, its expression is
$\mathcal{F}(T, \phi)=\frac{T^{4}}{2 \pi^{2}}\left[J_{B}\left(\frac{m_{0}(\phi)^{2}}{T^{2}}\right)-n_{F} J_{F}\left(\frac{m_{\psi}(\phi)^{2}}{T^{2}}\right)\right]$,

where the functions $J_{B}$ and $J_{F}$ are defined as

$$
J_{B}\left(\frac{m^{2}}{T^{2}}\right)=\int_{0}^{+\infty} d u u^{2} \log \left(1 \mp e^{-\sqrt{u^{2}+m^{2} / T^{2}}}\right) .
$$

At high temperature, these quantities can be expanded as

$$
\begin{aligned}
J_{B}\left(\frac{m^{2}}{T^{2}}\right)= & -\frac{\pi^{4}}{45}+\frac{\pi^{2}}{12} \frac{m^{2}}{T^{2}}-\frac{\pi}{6}\left(\frac{m^{2}}{T^{2}}\right)^{\frac{3}{2}} \\
& -\frac{1}{32} \frac{m^{4}}{T^{4}} \log \frac{m^{2}}{16 \alpha T^{2}}+\mathcal{O}\left(\frac{m^{6}}{T^{6}}\right), \\
J_{F}\left(\frac{m^{2}}{T^{2}}\right)= & \frac{7 \pi^{4}}{360}-\frac{\pi^{2}}{24} \frac{m^{2}}{T^{2}}-\frac{1}{32} \frac{m^{4}}{T^{4}} \log \frac{m^{2}}{\alpha T^{2}}+\mathcal{O}\left(\frac{m^{6}}{T^{6}}\right),
\end{aligned}
$$

where $\alpha=\pi^{2} \exp \left(3 / 2-2 \gamma_{\mathrm{E}}\right)$ and $\gamma_{\mathrm{E}}$ is the EulerMascheroni constant. Note that the $m_{0}^{4} \log m_{0}^{2}$ and $m_{\psi}^{4} \log m_{\psi}^{2}$ terms of the zero-temperature potential and free energy in Eqs. (7), (9), and (11) cancel exactly. However, comparing the expansions of $J_{B}$ and $J_{F}$, one sees that the free energy of the bosonic particles $\phi$ contains an extra source of nonanalyticity, namely, the term in $\left(m_{0}^{2}\right)^{\frac{3}{2}}$. Therefore, the one-loop thermal effective potential is complex when $m_{0}(\phi)^{2}<0$. In some cases, imaginary parts in effective potentials are associated with physical phase transitions [83]. However, in the present case, the nonanalytic term arises because thermal higher loop corrections that are of the same order of magnitude have been omitted. It turns out that the high-temperature quantum corrections are dominated by the so-called ring (or daisy) diagrams, with an arbitrary number of loops [84,85]. In practice, the resummation of the high-temperature limit of the ring diagrams amounts to adding a contribution to the one-loop potential [86],

$$
\mathcal{V}_{\text {eff }}^{\text {th }}(T, \phi) \equiv \mathcal{V}_{1-\text { loop }}^{\text {th }}(T, \phi)+\mathcal{V}_{\text {ring }}^{\text {th }}(T, \phi),
$$

where

$\mathcal{V}_{\text {ring }}^{\text {th }}(T, \phi)=\frac{T}{12 \pi}\left[\left(m_{0}(\phi)^{2}\right)^{\frac{3}{2}}-\left(m_{0}(\phi)^{2}+\Pi_{\phi}(T)\right)^{\frac{3}{2}}\right]$.

In the above expression, the shift of $m_{0}^{2}$ is the so-called Debye mass squared

$$
\Pi_{\phi}(T)=\frac{T^{2}}{24}\left(\lambda+n_{F} y^{2}\right),
$$

which is the dominant temperature contribution of $\partial^{2} \mathcal{F} /(\partial \phi)^{2}$. As a result, the pathological contributions 
$\left(m_{0}^{2}\right)^{\frac{3}{2}}$ of the one-loop and ring diagrams cancel exactly, while the remaining term $\left(m_{0}^{2}+\Pi_{\phi}\right)^{\frac{3}{2}}$ in $\mathcal{V}_{\text {ring }}^{\text {th }}$ is real.

Even if this is not necessary, we neglect from now on for the sake of simplicity all terms $T^{4} \times \mathcal{O}\left(\mathrm{m}^{6} / T^{6}\right)$ arising in the high-temperature expansions of the one-loop free energy density $\mathcal{F}$ and ring contribution $\mathcal{V}_{\text {ring }}^{\text {th }}$. In order to write the thermal effective potential in a suggestive way, we find it convenient to set the renormalization scale $Q$ in terms of a critical temperature,

$$
\begin{aligned}
Q & =\pi e^{-\gamma_{\mathrm{E}}} T_{c}, \\
T_{c} & =\frac{2 \sqrt{6} \mu}{\sqrt{\lambda+n_{F} y^{2}}} \sqrt{\frac{1-\frac{\sqrt{6}}{8 \pi} \xi+\frac{\log 2}{8 \pi^{2}} \lambda}{1-\frac{\sqrt{6}}{4 \pi} \xi}},
\end{aligned}
$$

where we have defined

$$
\xi \equiv \frac{\lambda}{\sqrt{\lambda+n_{F} y^{2}}} \in(\lambda, \sqrt{\lambda}) .
$$

Expressing all dependences in $T$ with a new variable $x$,

$$
x=\frac{T_{c}}{T},
$$

the effective potential at finite temperature reduces to

$$
\mathcal{V}_{\mathrm{eff}}^{\mathrm{th}}(x, \phi)=\mathcal{V}_{0}(x)-\frac{\mu_{\mathrm{eff}}(x)^{2}}{2} \phi^{2}+\frac{\lambda_{\mathrm{eff}}(x)}{4 !} \phi^{4}
$$

In this expression, $\mathcal{V}_{0}$ depends only on the temperature, while $\mu_{\text {eff }}^{2}$ is an effective mass term,

$$
\begin{aligned}
\mu_{\mathrm{eff}}(x)^{2}= & \mu^{2}\left[\left(1-\frac{\sqrt{6}}{8 \pi} \xi+\frac{\log 2}{8 \pi^{2}} \lambda\right)\left(1-\frac{1}{x^{2}}\right)\right. \\
& \left.-\frac{\lambda}{16 \pi^{2}} \log x\right],
\end{aligned}
$$

and $\lambda_{\text {eff }}$ is an effective self-coupling,

$$
\begin{aligned}
\lambda_{\text {eff }}(x)= & \lambda\left(1-\frac{3 \sqrt{6}}{8 \pi} \xi+\frac{3 \log 2}{8 \pi^{2}} \lambda\right) \\
& +\frac{3}{16 \pi^{2}}\left(4 n_{F} y^{4}-\lambda^{2}\right) \log x .
\end{aligned}
$$

The mass term $\mu_{\text {eff }}(x)^{2}$ increases from negative values at $x \ll 1$, up to a positive maximum at $x \simeq 4 \pi \sqrt{2 / \lambda}$, and it vanishes at $x=1 .^{2}$ Therefore, the field $\phi$ acquires thermal corrections to its mass, and a phase transition takes place at

\footnotetext{
${ }^{2}$ It is also positive between $x=1$ and the extremely high formal value $x \simeq e^{16 \pi^{2} / \lambda}$, which is far above the domain of validity of the thermal expression of the potential.
}

$x=1$, which justifies $T_{c}$ to be referred to as a critical temperature. The scalar $\phi$ is stabilized at 0 at higher temperature, while it condenses and follows adiabatically a temperature-dependent vev at lower temperature. We discuss in Sec. VI the validity of such a behavior.

\section{SPONTANEOUS FREEZE OUT OF DARK MATTER}

In this section, we explain how the fermionic darkmatter particles $\psi$ can freeze out, while the dark-scalar ones remain in thermal equilibrium with the SM bath. However, this is fully justified in Sec. VI, where we present specific examples of interactions $\mathcal{L}_{\text {int }}$ between dark sector and SM fields.

After the dark-matter particles $\psi$ freeze out at $T=T_{\mathrm{FO}}$, only $\phi$ remains eventually thermalized with the SM. The expression of the effective potential we considered so far is therefore valid up to $x_{\mathrm{FO}}=T_{c} / T_{\mathrm{FO}}$. The vev of $\phi$ reads $^{3}$

$$
x \leq 1:\langle\phi\rangle=0,
$$

$$
1 \leq x \leq x_{\mathrm{FO}}:\langle\phi\rangle=\mu_{\mathrm{eff}}(x) \sqrt{\frac{6}{\lambda_{\mathrm{eff}}(x)}},
$$

and the dark-scalar mass evolves accordingly like

$$
\begin{gathered}
x \leq 1: m_{\phi}(x)=\left|\mu_{\mathrm{eff}}(x)\right|, \\
1 \leq x \leq x_{\mathrm{FO}}: m_{\phi}(x)=\sqrt{2} \mu_{\mathrm{eff}}(x) .
\end{gathered}
$$

As a result, the fermionic dark-matter mass becomes effectively a function of the temperature,

$$
\begin{gathered}
x \leq 1: m_{\psi}(x)=0, \\
1 \leq x \leq x_{\mathrm{FO}}: m_{\psi}(x)=y \sqrt{\frac{3}{\lambda_{\mathrm{eff}}(x)}} m_{\phi}(x) .
\end{gathered}
$$

The mechanism which maintains dark-sector particles in thermal equilibrium before they freeze out can be of different natures. First $\psi$ remains thermalized with the $\mathrm{SM}$ as long as

$$
\text { (i) } H<n_{\psi}\left\langle\sigma_{\mathrm{SM} \leftrightarrow \psi \bar{\psi}} v\right\rangle,
$$

where $\left\langle\sigma_{\mathrm{SM} \psi \psi \bar{\psi}} v\right\rangle$ denotes the thermally averaged cross section of annihilation of $\psi$ into SM fields, $n_{\psi}$ denotes its number density, and $H$ is the Hubble parameter. Second, dark-matter particles can remain in thermal equilibrium with the dark scalar $\phi$, given that

\footnotetext{
${ }^{3}$ If at reheating temperature, $x=x_{\mathrm{RH}}$, the effective coupling is negative, $\lambda_{\text {eff }}\left(x_{\mathrm{RH}}\right)<0$, the vev $\langle\phi\rangle=0$ corresponds to a local minimum of the potential, which becomes global as $x$ approaches 1 from below.
} 


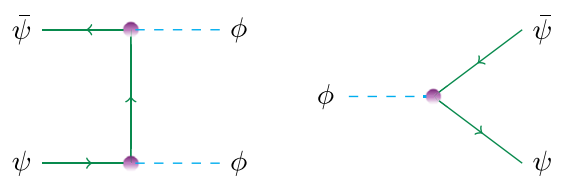

FIG. 1. Annihilation and decay processes contributing to thermal equilibrium in the dark sector.

$$
\text { (ii) } H<\Gamma_{\phi \rightarrow \psi \bar{\psi}} \quad \text { or } \quad H<n_{\psi}\left\langle\sigma_{\phi \phi \leftrightarrow \psi \bar{\psi}} v\right\rangle \text {, }
$$

where $\Gamma_{\phi \rightarrow \psi \psi \bar{\psi}}$ stands for the width of the (inverse) decay process $\phi \leftrightarrow \psi+\bar{\psi}$ and $\left\langle\sigma_{\phi \phi \leftrightarrow \psi \nu \bar{\psi}} v\right\rangle$ denotes the annihilation cross section of $\psi$ through a $t$-channel process (see Fig. 1). Finally, $\phi$ can thermalize with the SM as long as either the conditions (i) and (ii) are guaranteed simultaneously, or because interactions between $\phi$ and SM fields are present and such that

$$
\text { (iii) } H<n_{\phi}\left\langle\sigma_{\mathrm{SM} \phi \phi} v\right\rangle \text { or } H<\Gamma_{\phi \rightarrow \mathrm{SM}} \text {, }
$$

where $n_{\phi}$ is the number density of $\phi$, while $\left\langle\sigma_{\mathrm{SM} \phi \phi \phi} v\right\rangle$ and $\Gamma_{\phi \rightarrow \mathrm{SM}}$ are the annihilation cross section and decay width. In this last case (iii), $\psi$ does not necessarily have to interact with SM particles in order to thermalize with them, provided the condition (ii) is fulfilled. We depict the different Feynman diagrams which can lead to such interactions in Figs 1 and 2.

In Sec. VI we explicitly introduce interactions between the dark sector and the SM. We in particular focus on the simple case where

$$
\left\langle\sigma_{\mathrm{SM} \psi \psi \bar{\psi}} v\right\rangle \gg\left\langle\sigma_{\phi \phi \leftrightarrow \psi \bar{\psi}} v\right\rangle,
$$

such that the freeze out of dark matter is only driven by the contact interaction between $\psi$ and the SM fields, and not by the interactions taking place within the dark sector. We also work in a regime in which the dark scalar $\phi$ remains in thermal equilibrium with the SM while the dark-matter freeze out takes place. Therefore the condition (i) remains true until $x=x_{\mathrm{FO}}$. On the scalar side, the condition (ii) is satisfied when $x<1$ because dark-matter particles are massless and the inverse-decay process is kinematically allowed, whereas the condition (iii) is satisfied at later time, as we discuss in Sec. VI.
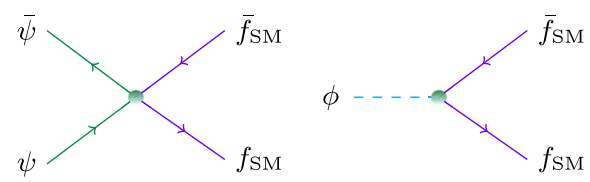

FIG. 2. Examples of annihilation and decay processes arising from the interaction Lagrangian $\mathcal{L}_{\text {int }}$ (see Sec. VI) which may contribute to the thermal equilibrium between $\psi, \phi$ and SM particles.
In principle, alternative scenarios, violating the condition of Eq. (27), in which $\psi$ remains in equilibrium with $\phi$ before freezing out, could be perfectly viable. This dark equilibrium could take place either together with the SM (if the $\phi \leftrightarrow S M$ interaction is strong enough) or in a dark equilibrium secluded from the SM bath (see, e.g., Refs. [87-89]). This possibility would however suppress the interaction between DM and SM fields and therefore be more challenging to detect experimentally.

\section{A. The spontaneous freeze out regime}

To proceed, let us define the ratio $\kappa=m_{\psi}\left(x_{\mathrm{FO}}\right) / T_{\mathrm{FO}}$. For $x_{\mathrm{FO}}>1$, we obtain from this definition the following expression,

$$
\begin{aligned}
x_{\mathrm{FO}}^{2}= & 1+\left(\frac{4 \kappa^{2}\left[\lambda+\frac{3}{16 \pi^{2}}\left(4 n_{F} y^{4}-\lambda^{2}\right) \log x_{\mathrm{FO}}\right]}{y^{2}\left(\lambda+n_{F} y^{2}\right)}\right. \\
& \left.+\frac{\lambda}{16 \pi^{2}} \log x_{\mathrm{FO}}\right)(1+\mathcal{O}(\xi)),
\end{aligned}
$$

which is valid in perturbative regime. In practice, the value of $\kappa$ depends on the explicit temperature dependency of the DM annihilation cross section into SM particles and is well known to be of order $\mathcal{O}(20-30)$ for masses in the $\mathrm{GeV}-\mathrm{TeV}$ range. It is in Sec. VI that we numerically evaluate the freeze out temperature, using explicit examples of interactions between $\psi$ and SM particles. From Eq. (28), we observe that different regions of the parameter space can be distinguished. For a large scalar self-interaction, as compared to the Yukawa interaction, we have

$$
\lambda \gg n_{F} y^{2} \Rightarrow x_{\mathrm{FO}} \simeq \mathcal{O}\left(\frac{2 \kappa}{y}\right) \gg \kappa .
$$

However, in the reversed case, we obtain

$\lambda \ll n_{F} y^{2} \Rightarrow x_{\mathrm{FO}} \simeq\left[1+\kappa^{2}\left(\frac{4 \lambda}{n_{F} y^{4}}+\frac{3}{\pi^{2}} \log x_{\mathrm{FO}}\right)\right]^{1 / 2}$,

which allows $x_{\mathrm{FO}}$ to be relatively smaller than $\kappa$ as long as $\lambda<y^{4}$. We can therefore identify two different regimes:

(i) When $x_{\mathrm{FO}} \gg \kappa$, the temperature at which darkmatter freezes out is much lower than the critical one, $T_{\mathrm{FO}} \ll T_{c}$. At such a low temperature, the masses $m_{\phi}\left(x_{\mathrm{FO}}\right)$ and $m_{\psi}\left(x_{\mathrm{FO}}\right)$ [see Eqs (22), (23) and (19), (20)] are close to their tree-level values,

$$
\begin{aligned}
1 \ll x \leq x_{\mathrm{FO}}: m_{\phi}(x) & \simeq m_{\phi}^{\text {tree }}, \\
m_{\psi}(x) & \simeq m_{\psi}^{\text {tree }} .
\end{aligned}
$$

Figure 3(a) shows the fermion mass $m_{\psi}$ (brown), scalar mass $m_{\phi}$ (red), and temperature (teal) as 
(a)

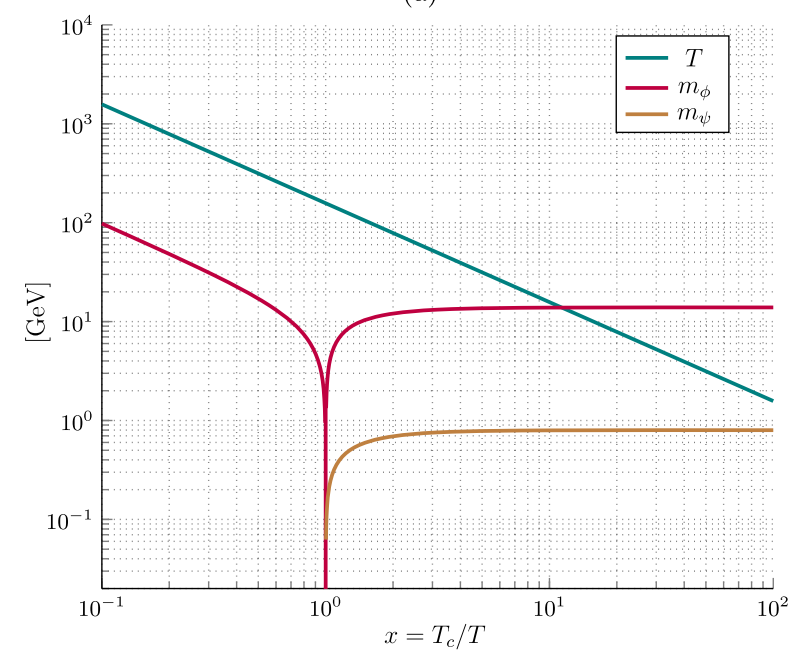

(b)

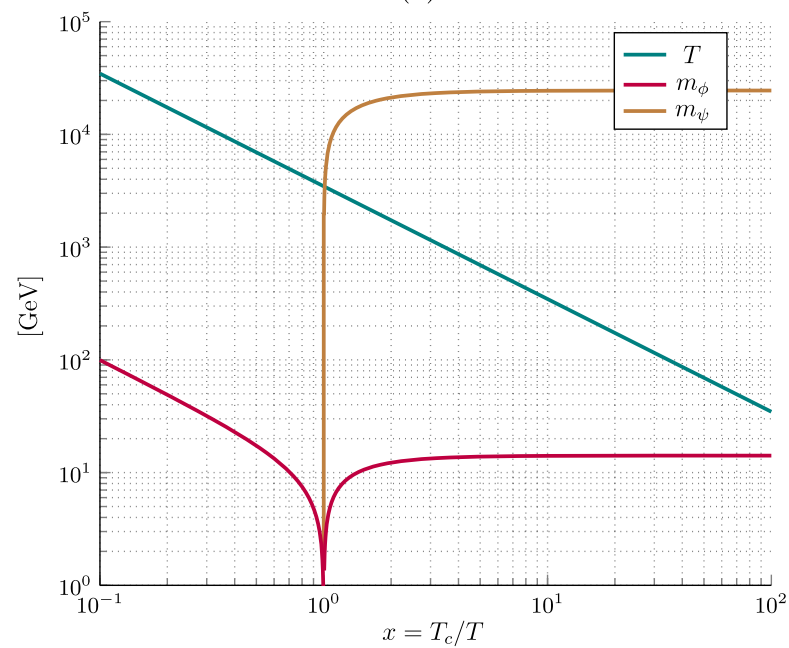

FIG. 3. Examples reproducing (a) the usual freeze out and (b) spontaneous freeze out scenarios in the case where $y=10^{-2}, \mu=$ $10 \mathrm{GeV}$ and, respectively, $\lambda=10^{3} y^{2}=0.1$ and $\lambda=10^{-2} y^{4}=10^{-10}$.

functions of $x$, in the illustrative case where $\mu=$ $10 \mathrm{GeV}, y=10^{-2}, n_{F}=2$, and $\lambda=10^{3} y^{2}=0.1$. Because $x_{\mathrm{FO}} \gg \kappa$, the temperature reaches $m_{\psi}$ when the mass of $\psi$ is already varying slowly, which corresponds to the usual thermal freeze out paradigm.

(ii) When $x_{\mathrm{FO}} \lesssim \kappa$, the freeze out takes place soon after the fermionic dark-matter particles start becoming massive. At this epoch, the evolutions of the masses are

$$
\begin{aligned}
& 1 \leq x \leq x_{\mathrm{FO}}: m_{\phi}(x) \simeq m_{\phi}^{\mathrm{tree}} \sqrt{1-\frac{1}{x^{2}}} \\
& m_{\psi}(x) \simeq y \mu \sqrt{\frac{6}{\lambda+\frac{3 n_{F}}{4 \pi^{2}} y^{4} \log x}} \sqrt{1-\frac{1}{x^{2}}} .
\end{aligned}
$$

Because shortly after the phase transition the derivative $d m_{\psi} / d x$ is very large, it is expected that the mass of the DM particles $\psi$ when they free out differs significantly from its final value today. In Fig. 3(b), we show the evolutions of the temperature and DM particle masses with respect to $x$ in this case. For $\mu=10 \mathrm{GeV}, y=10^{-2}, n_{F}=2$, and $\lambda=10^{-2} y^{4}=10^{-10}$, one can see that $m_{\psi}$ becomes larger than the temperature soon after the phase transition takes place, suggesting that the DM particles $\psi$ might indeed decouple while their mass still varies significantly. This illustrates the case we are mostly interested in this work, and that we refer to as spontaneous freeze out.

To summarize, we may have two different freeze out scenarios,

$$
\begin{aligned}
& x_{\mathrm{FO}} \gg \kappa: \text { constant-mass freeze out, } \\
& x_{\mathrm{FO}} \simeq \kappa: \text { spontaneous freeze out. }
\end{aligned}
$$

Notice that in the SFO case, we discard the possibility of having $1 \lesssim x_{\mathrm{FO}}<\kappa$ since, as we see in the next section, demanding the existence of a stable minimum of the potential at late time, when the temperature vanishes, imposes a lower bound of order $\kappa$ on $x_{\mathrm{FO}}$. In practice, this condition leads to a lower bound of order $0.03 n_{F}$ on $\lambda / y^{4}$ [see Eq. (45)].

\section{DYNAMICS OF THE SCALAR FIELD $\phi$ AFTER FREEZE OUT OF $\psi$}

In the present section, we are interested in the late time dynamics of the scalar $\phi$, when the temperature is well below its zero-temperature mass, and its effective potential can be approximated by its zero-temperature expression. Because the mass of the nonrelativistic DM particles $\psi$ of the relic density is $\phi$ dependent, the dust also sources the scalar potential in a way to be figured out. In total, the equation of motion of the scalar $\phi$ can be written as

$$
\ddot{\phi}+\left(\Gamma_{\phi}+3 H\right) \dot{\phi}=-\frac{d \mathcal{V}_{\text {eff }}}{d \phi}-\frac{d \mathcal{V}_{\text {dust }}}{d \phi},
$$

where the source $\mathcal{V}_{\text {dust }}$ arises from the dust, while the contribution $\mathcal{V}_{\text {eff }}$ is the zero-temperature effective potential of $\phi$. Note that we also include the friction term arising from the decay of $\phi$ into lighter fields. At one loop, the potential $\mathcal{V}_{\text {eff }}$ involves the Coleman-Weinberg and counterterm contribution of Eq. (7),

$$
\mathcal{V}_{\text {eff }}(\phi)=\mathcal{V}_{\text {tree }}(\phi)+\mathcal{V}_{\mathrm{CW}}(\phi)+\mathcal{V}_{\text {dark }}^{\text {c.t. }}(\phi) \text {. }
$$

In order to find $\mathcal{V}_{\text {dust }}$, a possible approach is to consider that beside the Einstein action coupled to the fundamental DM fields $\phi, \psi$, and to the SM degrees of freedom, we add an action for describing the motion of $N_{\psi}$ noninteracting 
particles of dust $\psi$. Each of them has coordinates $X_{i}^{\mu}\left(\tau_{i}\right)$, where $\tau_{i}$ is an arbitrary parameter along the world-line trajectory of particle $i$. Because the action of the fields $X_{i}^{\mu}\left(\tau_{i}\right)$ must be independent of the parametrization, it can be chosen to be proportional to the proper time along the trajectory multiplied by the mass,

$$
S_{\mathrm{dust}}=-\sum_{i} \int d \tau_{i} y\left|\phi\left(X_{i}\right)\right| \sqrt{g_{\mu \nu}\left(X_{i}\right) \frac{d X_{i}^{\mu}}{d \tau_{i}} \frac{d X_{i}^{\nu}}{d \tau_{i}}},
$$

where $g_{\mu \nu}$ is the spacetime metric. Varying $S_{\text {dust }}$ with respect to $g_{\mu \nu}$ and $\phi$, it is possible to derive the energy density $\rho_{\text {dust }}$, the pressure $P_{\text {dust }}$, and the contribution $\mathcal{V}_{\text {dust }}$ to the potential that arise from the distribution of DM particles $\psi$ [90,91], assuming that the latter is compatible with spacetime homogeneity and isotropy of the Universe at large enough scale.

However, for our purpose it is enough to multiply by $\dot{\phi}$ the equation of motion of $\phi$, Eq. (34), to obtain

$$
\dot{\rho}_{\phi}+\left(\Gamma_{\phi}+3 H\right)\left(\rho_{\phi}+P_{\phi}\right)=-\dot{\phi} \frac{d \mathcal{V}_{\text {dust }}}{d \phi},
$$

where $\rho_{\phi}, P_{\phi}$ are the gravitational sources associated with the dark scalar,

$$
\rho_{\phi}=\frac{1}{2} \dot{\phi}^{2}+\mathcal{V}_{\text {eff }}(\phi), \quad P_{\phi}=\frac{1}{2} \dot{\phi}^{2}-\mathcal{V}_{\text {eff }}(\phi) .
$$

Denoting $\rho_{\text {rad }}, P_{\text {rad }}$ the energy density and pressure of the visible sector, we also have [92]

$$
\dot{\rho}_{\text {rad }}+3 H\left(\rho_{\text {rad }}+P_{\text {rad }}\right)=\Gamma_{\phi}\left(\rho_{\phi}+P_{\phi}\right) .
$$

Note that we considered here that the scalar $\phi$ decays exclusively into SM particles since we consider in Sec. VI the region of the parameter space where the mass of $\psi$ is always larger than the mass of $\phi$. Combining Eqs (37), (39), and the conservation of the stress-energy tensor associated with the total system composed of the scalar $\phi$, the massless SM, and the dust, we obtain [73-75,93]

$$
\dot{\rho}_{\text {dust }}+3 H\left(\rho_{\text {dust }}+P_{\text {dust }}\right)=\dot{\phi} \frac{d \mathcal{V}_{\text {dust }}}{d \phi} .
$$

We are interested in the case where the relic dark-matter particles $\psi$ are nonrelativistic. Assuming for simplicity the limit case of vanishing particle velocities, the DM fluid is pressureless, while its energy density originates only from invariant mass,

$$
\rho_{\text {dust }}=n_{\psi} y|\phi|, \quad P_{\text {dust }}=0 .
$$

Using Eq. (40) and the fact that $n_{\psi}=N_{\psi} / a^{3}$, where $a$ is the scale factor of the Universe, we obtain

$$
\frac{d \mathcal{V}_{\text {dust }}}{d \phi}=\operatorname{sign}(\phi) y n_{\psi} .
$$

The effect of dust on the dynamics of the scalar $\phi$ after the fermionic DM freezes out is however minor, due to the suppression factor $1 / a^{3}$ in $n_{\psi}$. To see this qualitatively, we may neglect the Coleman-Weinberg and counterterm contributions to the scalar effective potential, and study the minima of $\mathcal{V}_{\text {tree }}+\mathcal{V}_{\text {dust }}$. This potential admits a local minimum at some $\langle\phi\rangle>\mu \sqrt{2 / \lambda}$ when

$$
n_{\psi}<n_{c}=\frac{2 \sqrt{2}}{3} \frac{\mu^{3}}{y \sqrt{\lambda}} .
$$

Thanks to the expansion of the Universe, or if the DM particle $\psi$ is sufficiently heavy (and its number density small enough), the above condition always ends up satisfied and $\langle\phi\rangle$ can converge to the tree-level value $\langle\phi\rangle_{\text {tree }}$, given in Eq. (5). Note however that, in principle, the backreaction of the dust might destabilize the vacuum $\langle\phi\rangle>0$, right after the freeze out takes place. This would lead $\phi$ to decrease and possibly allow the dark-matter particles $\psi$ to rethermalize. However, such a behavior would be contradictory with the hypothesis that dark matter is nonrelativistic while estimating the backreaction. Therefore, a more thorough treatment of this possibility in the presence of a quasirelativistic velocity distribution would be required. We leave such a dedicated study for future work.

Neglecting this backreaction of the relic density, another source of destabilization of the vacuum $\langle\phi\rangle>0$ may arise from the Coleman-Weinberg and counterterm contributions to the effective potential. In the SFO regime, corresponding to the limit of small self-coupling $\lambda$ as compared to $y^{4}$, one can understand this point by neglecting the contribution to the Coleman-Weinberg potential arising from the scalar sector. The analytic expression of the vev of $\phi$ that minimizes the potential is then found to be

$$
\langle\phi\rangle \simeq \frac{4 \pi \mu}{y^{2} \sqrt{-n_{F} W\left(-\frac{16 \pi^{2} \mu^{2} \exp \left(-1-8 \pi^{2} \lambda / 3 n_{F} y^{4}\right)}{n_{F} Q^{2} y^{2}}\right)}},
$$

where $W(z)$ stands for the product logarithm (or Lambert $W$ function). Imposing the above value to be real amounts to demanding the argument of $W(z)$ to be larger than $-1 / e$, which in turn leads to the condition

$$
\frac{\lambda}{y^{4}}>\frac{3 n_{F}}{8 \pi^{2}}\left(\log \frac{2}{3}+2 \gamma_{\mathrm{E}}\right) \simeq 0.03 n_{F} .
$$

In non-SFO cases, larger values of the self-coupling $\lambda$ always imply the existence of a local or global minimum at some $\langle\phi\rangle_{0}>0$. Therefore, as long as the bound of Eq. (45) is satisfied, the vev of $\phi$ converges towards the minimum of the potential at zero temperature and zero 
backreaction of the dust, while the mass $m_{\psi}$ approaches its final constant value,

$$
m_{\psi}^{0}=y\langle\phi\rangle_{0}
$$

\section{INTERACTION WITH THE STANDARD-MODEL BATH}

In the previous sections, we have described the dynamics of a scalar field living in a thermal potential. We have, in particular, assumed that the dark-matter particles $\psi$ and the dark scalar $\phi$ were maintained in thermal equilibrium with the standard model before the spontaneous freeze out mechanism takes place. However, we have not specified yet in which way the dark sector interacts with standardmodel particles. In this section we explicitly introduce such interactions, perform a numerical scan, and derive important constraints on the parameter space.

Although one could consider a complete set of dimensionsix operators for describing the annihilation of the darkmatter particles $\psi$ into SM fermions, we consider in this paper only spin-independent interactions as an educational toy example and leave a more systematic study for future work. Therefore, we focus on the two simplest operators of this kind leading to $s$-wave and $p$-wave processes for darkmatter annihilation into $\mathrm{SM}^{4}$ fermions $f$, which are respectively through vector $(\mathrm{V})$ and scalar $(\mathrm{S})$ operators

$$
\mathcal{O}_{V}=\bar{\psi} \gamma_{\mu} \psi \bar{f} \gamma^{\mu} f \quad \text { and } \quad \mathcal{O}_{S}=\bar{\psi} \psi \bar{\psi} f
$$

Introducing the couplings $G_{V}$ and $G_{S}$, one can write the associated cross sections of dark-matter annihilation $\psi \bar{\psi} \rightarrow f \bar{f}$ as

$$
\begin{aligned}
\sigma_{V}= & \frac{G_{V}^{2}}{32 \pi} \sqrt{\frac{s-4 m_{f}^{2}}{s-4 m_{\psi}^{2}}} \\
& \times\left(\frac{\left(s-4 m_{\psi}^{2}\right)\left(s-4 m_{f}^{2}\right)}{3 s}+4\left(m_{\psi}^{2}+m_{f}^{2}\right)+s\right), \\
\sigma_{S}= & \frac{G_{S}^{2}}{32 \pi s} \sqrt{\frac{s-4 m_{f}^{2}}{s-4 m_{\psi}^{2}}}\left(s-4 m_{\psi}^{2}\right)\left(s-4 m_{f}^{2}\right) .
\end{aligned}
$$

Assuming that the mass of the fermion $f$ is much lighter than the dark-matter particle in the range $1<x \leq x_{\mathrm{FO}}$, and using the fact that the relative velocity of thermalized darkmatter particles satisfies $\left\langle v^{2}\right\rangle=6 T / m_{\psi}$, one can express the thermally averaged cross section of annihilation as

\footnotetext{
${ }^{4}$ Note that, in principle, the fermion $f$ may not be part of the standard-model spectrum, as long as the interactions with it are sufficient to keep $f$ in equilibrium with the SM bath until dark matter freezes out.
}

$$
\begin{aligned}
\langle\sigma v\rangle_{V} & \simeq \frac{G_{V}^{2}}{2 \pi}\left(1+\frac{x^{-1} T_{c}}{m_{\psi}(x)}\right) m_{\psi}^{2}(x), \\
\langle\sigma v\rangle_{S} & \simeq \frac{3 G_{S}^{2}}{8 \pi} x^{-1} T_{c} m_{\psi}(x) .
\end{aligned}
$$

\section{A. Relic density}

The evolution of the dark-matter number density $n_{\psi}$ can be described by the Boltzmann equation, which we express in terms of the yield $Y_{\psi}=n_{\psi} / s$,

$$
\frac{\mathrm{d} Y_{\psi}}{d x}=\frac{\langle\sigma v\rangle s}{x H}\left(Y_{\psi, \mathrm{eq}}^{2}-Y_{\psi}^{2}\right) .
$$

In this equation, $s$ is the entropy density of the Universe and $Y_{\psi \text {,eq }}=n_{\psi \text {,eq }} / s$ is the value of the yield when $\psi$ follows a Boltzmann equilibrium. After dark-matter particles and antiparticles freeze out, the relic density of dark matter is given by

$$
\Omega h^{2}=n_{F} \frac{m_{\psi}\left(x_{0}\right) s_{0}}{6 H_{0}^{2} M_{\mathrm{P}}^{2}} Y_{\psi}^{0},
$$

where indices " 0 " express the fact that the different quantities are evaluated at the present time, where $T=T_{0} \simeq 2.4 \times 10^{-4} \mathrm{eV}$. The entropy density of the Universe is therefore given by $s_{0}=2.1 \times 10^{-38} \mathrm{GeV}^{3}$, the Hubble parameter is $H_{0} \simeq 71 \mathrm{~km} \cdot \mathrm{s}^{-1} \cdot \mathrm{Mpc}^{-1}, h \equiv$ $H_{0} /\left(100 \mathrm{~km} \cdot \mathrm{s}^{-1} \cdot \mathrm{Mpc}^{-1}\right)$, and the Planck mass is denoted by $M_{\mathrm{P}}=1.22 \times 10^{19} \mathrm{GeV}$.

\section{B. Numerical results}

Before presenting our numerical results, let us make a few comments on what is expected in our scenario of spontaneous freeze out, as compared to the usual case of a constant-mass dark matter particle.

(i) For a given dark-matter mass $m_{\psi}^{0}$ and relic density at present time, the dark-matter yield at freeze out $Y_{\psi}^{\mathrm{FO}}$ is fixed.

(ii) The dark-matter relative velocity at freeze out $\left\langle v^{2}\right\rangle \sim T_{\mathrm{FO}} / m_{\psi}=\kappa^{-1}$ is essentially model independent. Because in our scenario $m_{\psi}\left(x_{\mathrm{FO}}\right)<m_{\psi}^{0}$, we expect the freeze out temperature in the SFO case to be lower than in the constant-mass standard WIMP scenario.

(iii) At freeze out, the condition $n_{\psi}^{\mathrm{FO}}\langle\sigma v\rangle_{\mathrm{FO}}=H_{\mathrm{FO}}$ can be expressed as

$$
Y_{\psi}^{\mathrm{FO}}\langle\sigma v\rangle_{\mathrm{FO}} \propto T_{\mathrm{FO}}^{-1} .
$$

Therefore the dark-matter annihilation cross section at freeze out $\sigma_{\mathrm{FO}}$ in the $\mathrm{SFO}$ case is larger than in the constant-mass paradigm. 
(iv) In our benchmark models, the cross sections of Eq. (49) evolve after freeze out as

$$
\begin{aligned}
\langle\sigma v\rangle_{V} & \simeq \frac{G_{V}^{2}}{2 \pi}\left(1+\frac{v^{2}}{6}\right) m_{\psi}^{2}(x), \\
\langle\sigma v\rangle_{S} & \simeq \frac{3 G_{S}^{2}}{48 \pi} v^{2} m_{\psi}(x) .
\end{aligned}
$$

Because in the SFO case the dark-matter particle mass increases with time, the ratio between the annihilation cross section in our scenario as compared to the usual WIMP case increases from the time of freeze out to present time, where the DM velocity $v \simeq 200 \mathrm{~km} \cdot \mathrm{s}^{-1}$ is model independent.

Note that if we had considered different operators than those chosen in Eq. (47), the dependency of the annihilation cross section with the DM mass would change. In particular, lower dimensional operators might lead to situations where $\langle\sigma v\rangle$ is either invariant or decreasing with the DM mass. If that is the case the effect described in the previous paragraph might be balanced by the evolution of the cross section between the time of freeze out and today.

In Figs. 4 and 5 we present our numerical results for the two benchmark operators $\mathcal{O}_{V}$ and $\mathcal{O}_{S}$. In both figures, we scan over the whole parameter space $\{\mu, \lambda, y\}$ in the case of a Majorana fermion $\left(n_{F}=2\right)$ and compute the annihilation cross section (and therefore the value of the coupling $G_{V, S}$ ) necessary to obtain the correct relic abundance of dark matter $\Omega h^{2} \simeq 0.12$. In particular, we focus on the case of a Yukawa coupling in the range $y \in[0.001,0.1]$ and values of $\lambda$ containing the SFO regime $\lambda=\left[0.03 n_{F} y^{4}, 16 y^{4}\right]$.
In order to scan over the DM mass, the parameter $\mu$ is varied over the range [50 MeV, $5 \mathrm{TeV}]$.

In both cases our findings are very similar and in very good agreement with the qualitative predictions that we have just developed about our SFO scenario. Indeed one can clearly see that data points that lead to low values of the ratio $x_{\mathrm{FO}} / \kappa$, and therefore correspond to the SFO case [as defined in Eq. (33)] show an annihilation cross section that can be significantly larger than in the usual freeze out scenario by more than 1 order of magnitude. As a matter of fact, because the ratio $\lambda / y^{4}$ admits a lower bound given in Eq. (45), it can be seen from Eq. (30) that the ratio $x_{\mathrm{FO}} / \kappa$ cannot be arbitrarily small. Therefore, the increase of the annihilation cross section cannot be arbitrarily large.

Another interesting feature of our scenario is that a fraction of the parameter space which is ruled out by the unitarity constraint in the constant-mass WIMP scenario remains allowed in the SFO case. Indeed, the unitarity bound that we obtain is now independent on the cross section of annihilation today and amounts to an upper bound on the DM mass which is unchanged as compared to the constant-mass case.

In Fig. 6 we also present the value of the ratio between the dark-matter particle mass at the time of freeze out and its value at present time.

\section{Phenomenological constraints}

As a direct consequence of such an increased cross section between the dark sector and the visible sector, models of thermal dark matter that were already experimentally ruled out in the usual constant-mass freeze out scenario turn out to be even more excluded in the SFO regime.

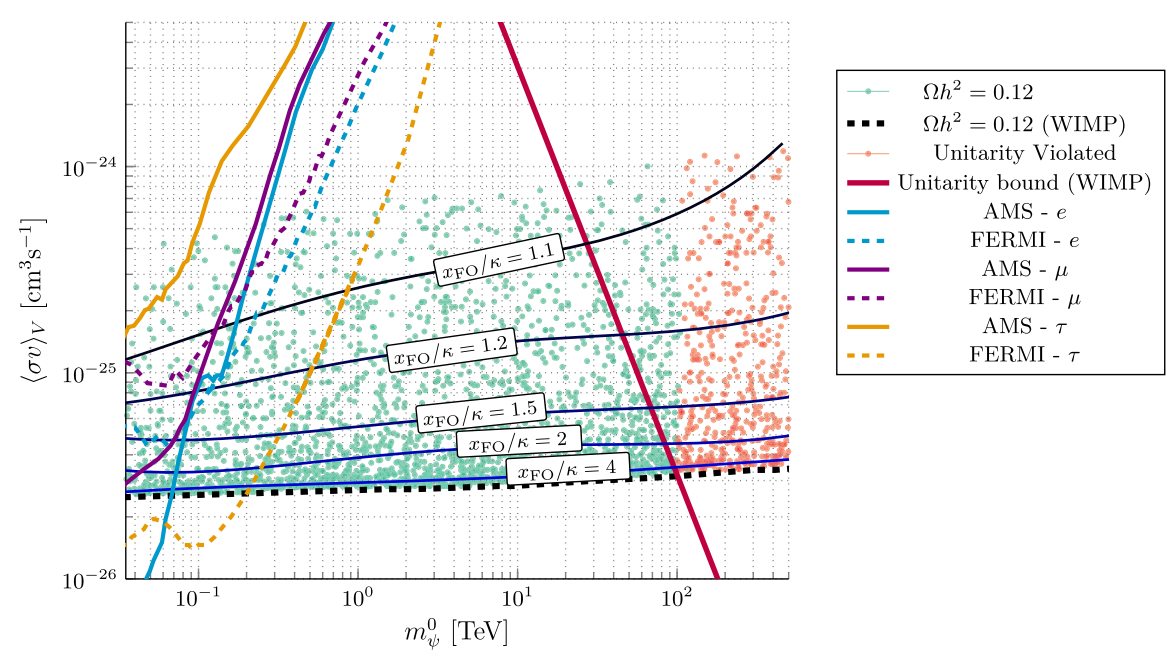

FIG. 4. Numerical results for the cross section of annihilation of dark-matter particles interacting with SM fermions via the operator $\mathcal{O}_{V}$. The plain red line indicates the standard WIMP unitarity bound, whereas the red dots stand for the points which violate unitarity in our scenario. Indirect-detection constraints on the annihilation cross section of a dark-matter candidate interacting with one single species of lepton are indicated. 


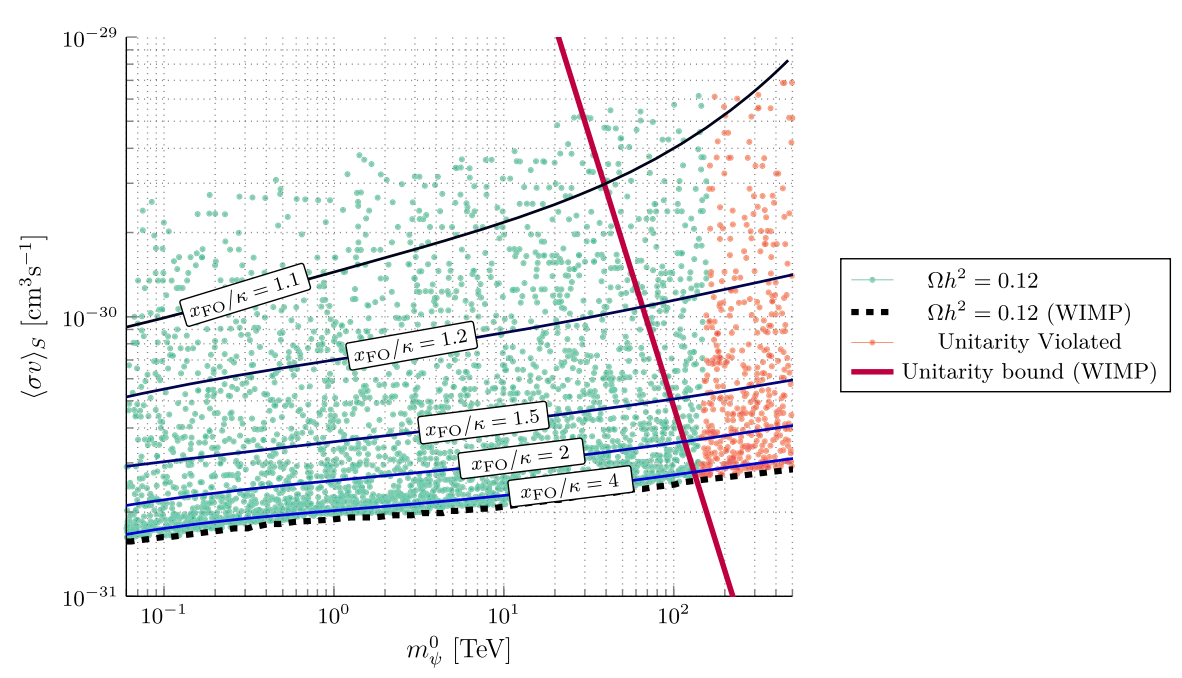

FIG. 5. Numerical results for the cross section of annihilation of dark-matter particles interacting with SM fermions via the operator $\mathcal{O}_{S}$. The plain red line indicates the standard WIMP unitarity bound, whereas the red dots stand for the points which violate unitarity in our scenario.

As a result, a model in which a dark-matter particle interacting with colored fermions via the vectorial effective operator $\mathcal{O}_{V}$ is totally ruled out by direct-detection constraints in our setup. Therefore, the only fermions with whom DM particles can interact in this case are charged leptons or neutrinos. Although the interaction of DM particles with electrons could induce some electron recoil, future direct-detection experiments are still far from constraining the region of parameter space which is of interest in our scenario.

In the case where DM interacts with colored particles through the scalar operator $\mathcal{O}_{S}$, direct-detection experiments

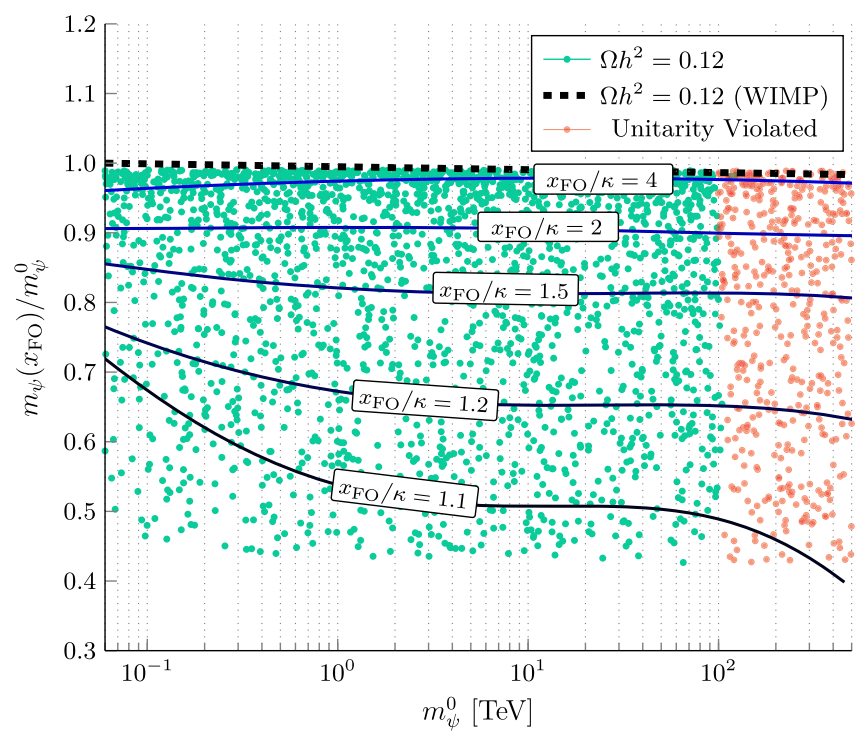

have ruled out a significant fraction of the parameter space, but heavy DM candidates could still, in principle, escape detection for masses above $\mathcal{O}(1) \mathrm{TeV}$. However, as we see in the next subsection, the existence of such an operator leads to an effective Yukawa coupling between the dark scalar $\phi$ and SM fermions. While $\phi$ acquires a large vev, such coupling can contribute dangerously to the mass of these fermions. As a matter of fact, it turns out that demanding such corrections to not overshoot the current experimental uncertainties on the quark masses eliminates all the data points which could escape the current direct detection limits set by Xenon1T [94]. Therefore we are to consider

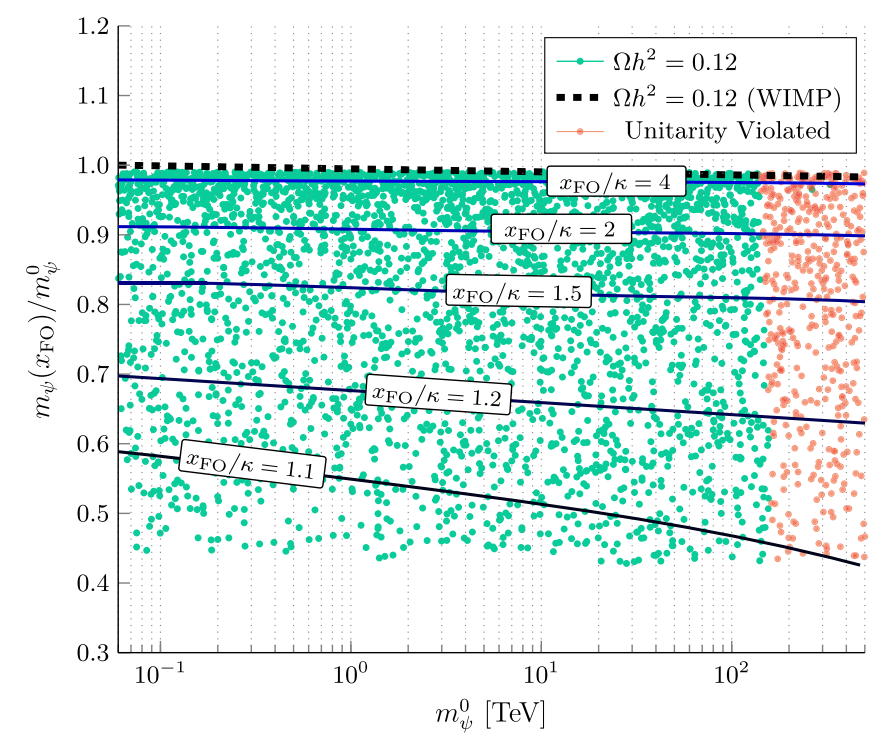

FIG. 6. Ratio between the values of the dark-matter particle mass at the time of freeze out and at present time for data points leading to the correct relic abundance of dark matter. The left panel corresponds to an $s$-wave annihilation cross section (operator $\mathcal{O}_{V}$ ), whereas the right panel stands for the $p$-wave annihilation cross section (operator $\mathcal{O}_{S}$ ). Red circles are associated with data points for which the annihilation cross sections violate unitarity at the time of freeze out. 
only in what follows that dark-matter particles interact with SM leptons.

\section{Indirect detection}

In the case of a velocity-suppressed cross section ( $p$ wave), the annihilation of dark-matter particles in the Galaxy today cannot lead to any visible signal under the form of cosmic rays, unless enhanced by long-range interactions (Sommerfeld enhancement). However, in the case of an $s$-wave annihilation process, there exists a plethora of constraints that can be used to set stringent limits on our parameter space. Indeed, for masses $\lesssim 10 \mathrm{GeV}$, the annihilation of dark-matter particles, even after they have frozen out, injects a fraction of energy that can distort significantly the cosmic microwave background [95], leading to severe constraints on the annihilation cross section of dark-matter particles. Moreover, above $10 \mathrm{GeV}$, the FERMI collaboration measuring the spectrum of dwarf spheroidal galaxies in the Milky Way [96,97], and the Alpha Magnetic Spectrometer (AMS) detecting cosmic rays set the most robust limits on dark-matter annihilation in the Galaxy $[98,99]$. In Fig. 4, we indicate the different constraints that have been derived in Ref. [100] for various possible leptonic final states. The fact that the annihilation cross section in the SFO case is significantly larger than in the usual constant-mass scenario leads to much more severe constraints. Therefore, heavy dark-matter candidates, which are unlikely to be detected in the near future in the usual constant-mass WIMP scenario, turn out to be very soon accessible to collaborations such as FERMI or AMS in the SFO case.

\section{Constraints on the scalar sector}

As we have anticipated in Sec. IV, throughout the Universe's history, the scalar field $\phi$ can have access to different decay channels: For $x<1$, we have seen that the fermionic dark-matter particles are massless and $\phi$ can decay at tree level, with the lifetime

$$
x<1:\left(\tau_{\phi}\right)^{-1}=\Gamma_{\phi \rightarrow \bar{\psi} \psi}=\frac{y^{2}}{8 \pi} m_{\phi}(x) .
$$

After the phase transition, when $\lambda \ll n_{F} y^{2}$, whether we are in the spontaneous freeze out case (where this condition is automatically satisfied) or not, the ratio of the masses in the dark sector can be expressed as follows:

$$
1 \leq x \leq x_{\mathrm{FO}}: \frac{m_{\psi}(x)^{2}}{m_{\phi}(x)^{2}} \simeq \frac{3 y^{2}}{\lambda+\frac{3 n_{F}}{4 \pi^{2}} y^{4} \log x} \gg 1 .
$$

As a result, in this region of parameter space, the decay and inverse decay $\phi \leftrightarrow \psi+\bar{\psi}$ are now kinematically forbidden. However, at the loop level, certain operators can lead to an effective decay of the dark scalar into SM particles, as

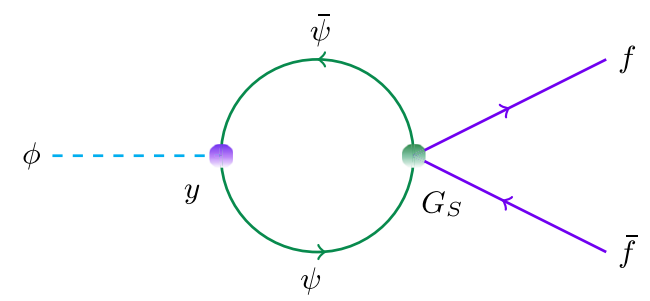

FIG. 7. Decay of the scalar $\phi$ into SM fermions via a loop of dark-matter particles.

depicted in Fig. 7. In the case of the scalar operator $\mathcal{O}_{S}$, the effective coupling induced by such a diagram is

$$
\mathcal{O}_{S}^{\mathrm{eff}} \sim\left(y \frac{G_{S} m_{\psi}^{2}}{16 \pi^{2}}\right) \phi \bar{f} f
$$

and it provides the scalar lifetime (for $x>1$ )

$$
\left(\tau_{\phi}^{S}\right)^{-1}=\Gamma_{\phi}^{S} \sim \frac{m_{\phi}}{8 \pi}\left(\frac{y G_{S} m_{\psi}^{2}}{16 \pi^{2}}\right)^{2}\left(1-\frac{4 m_{f}^{2}}{m_{\phi}^{2}}\right)^{3 / 2} .
$$

It is important to notice that the presence of the effective Yukawa coupling of Eq. (56) between the dark scalar $\phi$ and SM fermions can source a correction to the mass term of the latter after $\phi$ acquires a large vev. Since the dark-matter mass is given by $m_{\psi}=y\langle\phi\rangle$, it is clear from Eq. (56) that the corresponding correction to the mass of the SM fermion with whom the dark-matter particles $\psi$ interact, is of order

$$
\Delta m_{f} \sim G_{S} m_{\psi}^{3} .
$$

In practice, given the relatively large value of the coupling $G_{S}$ in the SFO case, we obtain a contribution to the mass of the SM fermion $f$ of order $\Delta m_{f}=\mathcal{O}\left(10^{-3}\right) m_{\psi}$. Given the extremely good accuracy with which the SM fermion masses are measured, the presence of such contribution imposes an upper bound on the dark-matter particle mass that depends on the details of the model considered.

However, in the case of the vectorial operator $\mathcal{O}_{V}$ such an effective coupling is forbidden. Therefore, unless adding contact interactions between $\phi$ and SM particles, the dark scalar could, in principle, be relatively stable on cosmological scales. The possibility that the dark scalar would be long lived after the phase transition takes place can have four major consequences.

(i) If the lifetime of the scalar field $\phi$ is longer than the age of the Universe, it could participate to the DM relic abundance together with the $\psi$ particles after freezing out from the thermal bath.

(ii) The coherent oscillations of $\langle\phi\rangle$ around the minimum of the potential when the temperature of the Universe has dropped below the mass of $\phi$ might contribute significantly to the matter abundance and overclose the Universe [101-103]. This problem, 
which is known as the Polonyi problem [104,105], has in particular been raised in the context of supergravity and string theory [106].

(iii) If the energy density of the scalar (either due to its coherent oscillations or to its relic density) comes to dominate the energy density of the Universe before the scalar decays into SM particles, the corresponding entropy injection into the visible bath is known to reduce the dark-matter relic abundance $[87,88,92]$. In that case, this effect has to be taken into account in the numerics.

(iv) Finally, if $\phi$ decays after the big bang nucleosynthesis takes place, it can destroy the predictions for the different atom density fractions in the Universe today.

For these different reasons, it is essential that the dark scalar possesses a significant decay width after the phase transition takes place. Therefore we assumed in our scenario that such a decay rate is present and does not lead the dark scalar to contribute to the relic abundance at all or to dominate the energy density of the Universe at any time.

As a matter of fact, whether the effective operator of Eq. (56) exists or not for a given interaction of dark-matter particles $\psi$ with the SM, it is expected that our dark scalar can mix with the SM scalar. Therefore, depending on the mixing of $\phi$ with the Higgs boson, the former can possess a significant decay width after the phase transition happens. This in turn can solve all the issues listed above. Note that in the case of the scalar operator we introduced in Eq. (56), a mixing of the scalar $\phi$ with the Higgs boson arises at the two-loop level. In any case, particular attention should be given to the scalar mass matrix and how the dark phase transition might affect the Higgs sector.

Finally, note that the t-channel annihilation of DM particles $\psi$ into scalars $\phi$ could, in principle, play an important role in the calculation of the DM relic density as long as it would dominate over the annihilation into SM fermions. However, in our scan, we have focused on a regime of parameters for which we have checked that this is not the case. Indeed the $t$-channel annihilation cross section scales like $y^{4} / m_{\psi}^{2}<10^{-4} / m_{\psi}^{2}$ in our scan, whereas the coupling $G_{S, V}$ turns out to be relatively large in our simulations, of order $\gtrsim 10^{-2} / \mathrm{m}_{\psi}^{2}$ leading to an annihilation cross section $\sigma v \simeq G_{V, S}^{2} m_{\psi}^{2} \gtrsim 10^{-4} / m_{\psi}^{2}$.

\section{E. Effect of the non-Adiabaticity}

Up to now, we have been assuming that the scalar field $\phi$ systematically tracks the minimum of its thermal potential in an adiabatic fashion. We have seen that such a tracking can force the freeze out temperature of dark matter to be lower than what one would infer from the usual constantmass WIMP scenario. We showed that this lowering of the freeze out temperature leads to a significantly larger

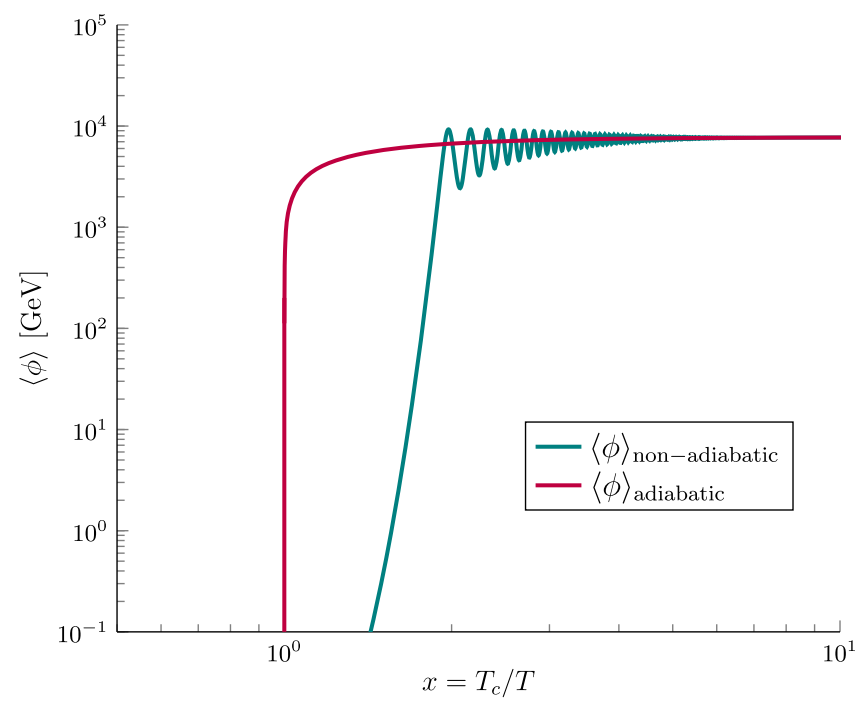

FIG. 8. Numerical simulation of the scalar field dynamics after the phase transition takes place for $y=10^{-2}, \lambda=10 y^{4}=10^{-7}$, $\mu=1 \mathrm{GeV}$ and including the scalar decay width $\Gamma_{\phi}=10^{-2} \mu$.

annihilation cross section of dark matter in order to accommodate the correct relic abundance.

In practice, when the phase transition takes place, the thermal mass of the scalar field is close to 0 , and it takes time before $\langle\phi\rangle$ falls and oscillates around the minimum of its potential. One can visualize this effect in Fig. 8 on a particular example. This leads the dark-matter mass to overshoot the Universe temperature at an even later time (i.e., lower temperature) than what we have been using in our numerical simulations. Therefore, the true value of $m_{\psi}\left(x_{\mathrm{FO}}\right)$ is expected to be lower than that we have described. In other words, the analysis that we performed throughout this work is relatively conservative and is likely to underestimate the effect that the SFO regime can have on the annihilation cross section of dark-matter particles, as well as on the increase of the DM mass between the freeze out time and today. Although we restrict ourself to an adiabatic description of the SFO mechanism in this work, we leave the thorough study of this effect in the presence of a nonadiabatic behavior of the scalar for future work.

\section{CONCLUSION}

In this paper, we have focused on the simple case in which a dark-matter fermionic particle acquires its mass from the spontaneous breaking of a global $\mathbb{Z}_{2}$ symmetry, and is in thermal equilibrium with the standard-model bath. We have computed the thermal corrections to the scalar potential that arise from the contribution of thermalized dark-sector particles to the free energy. The spontaneous breaking of the global $\mathbb{Z}_{2}$ symmetry is driven by a second order phase transition, similarly to what happens to the Higgs boson in the early Universe during the electroweak phase transition. We have demonstrated that the dynamics 
of such a phase transition, through which dark-matter particles spontaneously acquire mass, might interfere with the thermal freeze out mechanism. We have in particular identified an interesting region of the parameter space where the vacuum expectation value of the scalar field is large enough for the second order phase transition to enforce a spontaneous freeze out of dark-matter particles from the thermal bath. We have studied the phenomenology of our model in this regime in the simple case where darkmatter particles interact with SM fermions through spinindependent dimension-six operators. We have scanned over the parameter space in order to accommodate the relicdensity constraint, and we have compared our results to the most recent limits on dark-matter direct and indirect detection from XENON 1T, FERMI, and AMS, respectively, depending on whether DM particles interact with colored particles or leptons.

To put it in a nutshell, we have shown that the SFO regime enforces DM particles to decouple at a lower freeze out temperature than in the usual constant-mass models of WIMPs. Moreover, in the cases we have studied, the SFO regime favors an annihilation cross section of DM particles into SM states that is larger by more than 1 order of magnitude than that required in the WIMP paradigm, in order to obtain the correct relic abundance. This renders our model of SFO more experimentally testable than the constant-mass WIMP scenario. Last but not least, we have discussed the fact that the presence of nonadiabaticity in the way the dark scalar follows the minimum of its potential typically leads to an enhancement of the effect we have predicted.
As a conclusion, we emphasize that in every model of thermal dark matter in which the dark-matter particle mass is generated via spontaneous breaking of a high-energy global symmetry, one should, in principle, pay attention to the fact that thermal corrections to the scalar potential might affect significantly the freeze out mechanism. For a global $\mathbb{Z}_{2}$ symmetry breaking, we have shown that such corrections become significant if the quartic coupling $\lambda$ and the Yukawa coupling $y$ lie in the regime $\lambda \lesssim y^{4}$.

\section{ACKNOWLEDGMENTS}

The authors thank D. Chowdhury, E. Dudas, T. Hambye, F. Huang, K. Kaneta, D. Kim, F. Kling, M. Pierre, U. Reinosa, and D. Teresi for lively discussions and useful inputs during the realization of this work. L. H. also thanks G.-A. Soto for inspiring discussions throughout the realization of this work. The research activities of L. H. are supported in part by the Department of Energy under Grant No. DE-FG02-13ER41976 (de-sc0009913). The work of H. P. is partially supported by the Royal-Society/CNRS International Cost Share Grant No. IE160590. This work was partially performed at the Aspen Center for Physics, which is supported by National Science Foundation Grant No. PHY-1607611, as well as at the CERN Theory Department. This work was made possible by Institut Pascal at Université Paris-Saclay with the support of the P2I and SPU research departments and the P2IO Laboratory of Excellence (program "Investissements d'avenir" Grants No. ANR-11-IDEX-0003-01 ParisSaclay and No. ANR-10-LABX-0038), as well as the IPhT.
[1] L. Bergström, Rep. Prog. Phys. 63, 793 (2000).

[2] G. Bertone, D. Hooper, and J. Silk, Phys. Rep. 405, 279 (2005).

[3] G. Steigman, B. Dasgupta, and J. F. Beacom, Phys. Rev. D 86, 023506 (2012).

[4] C. Patrignani et al. (Particle Data Group), Chin. Phys. C 40, 100001 (2016).

[5] K. Griest and M. Kamionkowski, Phys. Rev. Lett. 64, 615 (1990).

[6] F. Richard, G. Arcadi, and Y. Mambrini, Eur. Phys. J. C 75, 171 (2015).

[7] L. M. Carpenter, A. Nelson, C. Shimmin, T. M. P. Tait, and D. Whiteson, Phys. Rev. D 87, 074005 (2013).

[8] L. Carpenter, A. DiFranzo, M. Mulhearn, C. Shimmin, S. Tulin, and D. Whiteson, Phys. Rev. D 89, 075017 (2014).

[9] A. A. Petrov and W. Shepherd, Phys. Lett. B 730, 178 (2014).

[10] N. F. Bell, J. B. Dent, A. J. Galea, T. D. Jacques, L. M. Krauss, and T. J. Weiler, Phys. Rev. D 86, 096011 (2012).
[11] N. F. Bell, Y. Cai, and R. K. Leane, J. Cosmol. Astropart. Phys. 01 (2016) 051.

[12] A. Birkedal, K. Matchev, and M. Perelstein, Phys. Rev. D 70, 077701 (2004).

[13] Y. Gershtein, F. Petriello, S. Quackenbush, and K. M. Zurek, Phys. Rev. D 78, 095002 (2008).

[14] J. Goodman, M. Ibe, A. Rajaraman, W. Shepherd, T. M. P. Tait, and H.-B. Yu, Phys. Rev. D 82, 116010 (2010).

[15] A. Crivellin, U. Haisch, and A. Hibbs, Phys. Rev. D 91, 074028 (2015).

[16] F. J. Petriello, S. Quackenbush, and K. M. Zurek, Phys. Rev. D 77, 115020 (2008).

[17] A. Berlin, T. Lin, and L.-T. Wang, J. High Energy Phys. 06 (2014) 078.

[18] T. Lin, E. W. Kolb, and L.-T. Wang, Phys. Rev. D 88, 063510 (2013).

[19] P. J. Fox, R. Harnik, J. Kopp, and Y. Tsai, Phys. Rev. D 85, 056011 (2012).

[20] N. F. Bell, Y. Cai, J. B. Dent, R. K. Leane, and T. J. Weiler, Phys. Rev. D 92, 053008 (2015). 
[21] Y. Bai, J. Bourbeau, and T. Lin, J. High Energy Phys. 06 (2015) 205.

[22] M. Autran, K. Bauer, T. Lin, and D. Whiteson, Phys. Rev. D 92, 035007 (2015).

[23] A. Gupta, R. Primulando, and P. Saraswat, J. High Energy Phys. 09 (2015) 079.

[24] K. Ghorbani and L. Khalkhali, J. Phys. G 44, 105004 (2017).

[25] D. Abercrombie et al., Phys. Dark Universe 26, 100371 (2019).

[26] M. Escudero, A. Berlin, D. Hooper, and M.-X. Lin, J. Cosmol. Astropart. Phys. 12 (2016) 029.

[27] C. Amole et al. (PICO Collaboration), Phys. Rev. D 93, 052014 (2016).

[28] C. Amole et al. (PICO Collaboration), Phys. Rev. D 93, 061101 (2016).

[29] C. Fu et al. (PandaX-II Collaboration), Phys. Rev. Lett. 118, 071301 (2017); 120, 049902(E) (2018).

[30] D. S. Akerib et al. (LUX Collaboration), Phys. Rev. Lett. 116, 161302 (2016).

[31] E. Aprile et al. (XENON100 Collaboration), Phys. Rev. D 94, 122001 (2016).

[32] E. Aprile et al. (XENON Collaboration), Phys. Rev. Lett. 119, 181301 (2017).

[33] X. Cui et al. (PandaX-II Collaboration), Phys. Rev. Lett. 119, 181302 (2017).

[34] D. S. Akerib et al. (LUX Collaboration), Phys. Rev. Lett. 118, 021303 (2017).

[35] M. Aaboud et al. (ATLAS Collaboration), Phys. Lett. B 763, 251 (2016).

[36] M. Aaboud et al. (ATLAS Collaboration), Phys. Lett. B 765, 11 (2017).

[37] M. Aaboud et al. (ATLAS Collaboration), Eur. Phys. J. C 77, 393 (2017).

[38] M. Aaboud et al. (ATLAS Collaboration), Phys. Rev. D 96, 112004 (2017).

[39] M. Aaboud et al. (ATLAS Collaboration), Phys. Rev. Lett. 119, 181804 (2017).

[40] M. Aaboud et al. (ATLAS Collaboration), Phys. Lett. B 776, 318 (2018).

[41] M. Aaboud et al. (ATLAS Collaboration), Eur. Phys. J. C 78, 18 (2018).

[42] M. Aaboud et al. (ATLAS Collaboration), J. High Energy Phys. 01 (2018) 126.

[43] A. M. Sirunyan et al. (CMS Collaboration), J. High Energy Phys. 03 (2017) 061; 09 (2017) 106(E).

[44] A. M. Sirunyan et al. (CMS Collaboration), J. High Energy Phys. 07 (2017) 014.

[45] A. M. Sirunyan et al. (CMS Collaboration), J. High Energy Phys. 10 (2017) 180.

[46] A. M. Sirunyan et al. (CMS Collaboration), Eur. Phys. J. C 77, 845 (2017).

[47] A. M. Sirunyan et al. (CMS Collaboration), J. High Energy Phys. 06 (2018) 027.

[48] A. M. Sirunyan et al. (CMS Collaboration), Eur. Phys. J. C 78, 789 (2018).

[49] H. Baer, K.-Y. Choi, J. E. Kim, and L. Roszkowski, Phys. Rep. 555, 1 (2015).

[50] L. Roszkowski, E. M. Sessolo, and S. Trojanowski, Rep. Prog. Phys. 81, 066201 (2018).
[51] M. Ackermann et al. (Fermi-LAT Collaboration), Phys. Rev. D 89, 042001 (2014).

[52] E. Dudas, L. Heurtier, Y. Mambrini, and B. Zaldivar, J. High Energy Phys. 11 (2013) 083.

[53] M. Cirelli, Proc. Sci., ICRC2015 (2016) 014 [arXiv:1511 $.02031]$.

[54] J. M. Gaskins, Contemp. Phys. 57, 496 (2016).

[55] P. J. Fox and E. Poppitz, Phys. Rev. D 79, 083528 (2009).

[56] X.-J. Bi, X.-G. He, and Q. Yuan, Phys. Lett. B 678, 168 (2009).

[57] T. Cohen and K. M. Zurek, Phys. Rev. Lett. 104, 101301 (2010).

[58] Y. G. Kim and K. Y. Lee, Phys. Rev. D 75, 115012 (2007).

[59] J. S. Hagelin, G. L. Kane, and S. Raby, Nucl. Phys. B241, 638 (1984).

[60] Y. G. Kim, K. Y. Lee, and S. Shin, J. High Energy Phys. 05 (2008) 100.

[61] M. Pospelov, A. Ritz, and M. B. Voloshin, Phys. Lett. B 662, 53 (2008).

[62] S. Tulin, H.-B. Yu, and K. M. Zurek, Phys. Rev. D 87, 115007 (2013).

[63] J. Shelton, S. L. Shapiro, and B. D. Fields, Phys. Rev. Lett. 115, 231302 (2015).

[64] J. A. Evans, S. Gori, and J. Shelton, J. High Energy Phys. 02 (2018) 100.

[65] E. W. Kolb, D. J. H. Chung, and A. Riotto, AIP Conf. Proc. 484, 91 (1999).

[66] R. Allahverdi, K. Dutta, and A. Maharana, J. Cosmol. Astropart. Phys. 10 (2018) 038.

[67] L. J. Hall, K. Jedamzik, J. March-Russell, and S. M. West, J. High Energy Phys. 03 (2010) 080.

[68] J. McDonald, Phys. Rev. Lett. 88, 091304 (2002).

[69] J. McDonald, J. Cosmol. Astropart. Phys. 08 (2016) 035.

[70] K. Kaneta, Y. Mambrini, and K. A. Olive, Phys. Rev. D 99, 063508 (2019).

[71] X. Chu, Y. Mambrini, J. Quevillon, and B. Zaldivar, J. Cosmol. Astropart. Phys. 01 (2014) 034.

[72] G. Bhattacharyya, M. Dutra, Y. Mambrini, and M. Pierre, Phys. Rev. D 98, 035038 (2018).

[73] G. W. Anderson and S. M. Carroll, in Proceedings, 1st International Conference on Particle Physics and the Early Universe (COSMO 1997), Ambleside, England, 1997 (World Scientific, Singapore, 1998), p. 581, http:// www.lancs.ac.uk/.

[74] R. Rosenfeld, Phys. Lett. B 624, 158 (2005).

[75] U. Franca and R. Rosenfeld, Phys. Rev. D 69, 063517 (2004).

[76] M. J. Baker, M. Breitbach, J. Kopp, and L. Mittnacht, J. High Energy Phys. 03 (2018) 114.

[77] M. J. Baker and L. Mittnacht, J. High Energy Phys. 05 (2019) 070.

[78] T. Hambye, A. Strumia, and D. Teresi, J. High Energy Phys. 08 (2018) 188.

[79] L. Darmé, A. Hryczuk, D. Karamitros, and L. Roszkowski, J. High Energy Phys. 11 (2019) 159.

[80] T. Coudarchet, L. Heurtier, and H. Partouche, J. High Energy Phys. 03 (2019) 117.

[81] Y. Nakai, N. Shiba, and M. Yamada, Phys. Rev. D 96, 123518 (2017).

[82] M. Quiros, in Proceedings, ICTP Summer School in HighEnergy Physics and Cosmology, Miramare, Trieste, Italy, 
1998 (World Scientific, Singapore, 1999), Vol. 15, p. 436, https://inspirehep.net/record/494058.

[83] E. J. Weinberg and A.-q. Wu, Phys. Rev. D 36, 2474 (1987).

[84] L. Dolan and R. Jackiw, Phys. Rev. D 9, 3320 (1974).

[85] M. E. Carrington, Phys. Rev. D 45, 2933 (1992).

[86] C. Delaunay, C. Grojean, and J. D. Wells, J. High Energy Phys. 04 (2008) 029.

[87] L. Heurtier and F. Huang, Phys. Rev. D 100, 043507 (2019).

[88] A. Berlin, D. Hooper, and G. Krnjaic, Phys. Rev. D 94, 095019 (2016).

[89] M. Cirelli, Y. Gouttenoire, K. Petraki, and F. Sala, J. Cosmol. Astropart. Phys. 02 (2019) 014.

[90] G. R. Farrar and P. J. E. Peebles, Astrophys. J. 604, 1 (2004).

[91] T. Damour and A. M. Polyakov, Nucl. Phys. B423, 532 (1994).

[92] E. W. Kolb and M. S. Turner, Front. Phys. 69, 1 (1990).

[93] M. B. Hoffman, arXiv:astro-ph/0307350.

[94] E. Aprile et al. (XENON Collaboration), Phys. Rev. Lett. 122, 141301 (2019).
[95] T. R. Slatyer, Phys. Rev. D 93, 023527 (2016).

[96] M. Ackermann et al. (Fermi-LAT Collaboration), Phys. Rev. Lett. 115, 231301 (2015).

[97] A. Albert et al. (Fermi-LAT and DES Collaboration), Astrophys. J. 834, 110 (2017).

[98] M. Aguilar et al. (AMS Collaboration), Phys. Rev. Lett. 113, 121102 (2014).

[99] L. Accardo et al. (AMS Collaboration), Phys. Rev. Lett. 113, 121101 (2014).

[100] R. K. Leane, T. R. Slatyer, J. F. Beacom, and K. C. Y. Ng, Phys. Rev. D 98, 023016 (2018).

[101] J. Preskill, M. B. Wise, and F. Wilczek, Phys. Lett. 120B, 127 (1983).

[102] L. F. Abbott and P. Sikivie, Phys. Lett. 120B, 133 (1983).

[103] M. Dine and W. Fischler, Phys. Lett. 120B, 137 (1983).

[104] G. D. Coughlan, W. Fischler, E. W. Kolb, S. Raby, and G. G. Ross, Phys. Lett. 131B, 59 (1983).

[105] J. R. Ellis, D. V. Nanopoulos, and M. Quiros, Phys. Lett. B 174, 176 (1986).

[106] B. de Carlos, J. A. Casas, F. Quevedo, and E. Roulet, Phys. Lett. B 318, 447 (1993). 